\title{
The Incidence of Potential Candidates for Total Disc Replacement among Lumbar and Cervical Fusion Patient Populations
}

\author{
Martin Quirno, Jeffrey A. Goldstein, John A. Bendo, Yong Kim, Jeffrey M. Spivak \\ Department of Orthopaedic Surgery, NYU Hospital for Joint Diseases, New York, NY 10003, USA
}

\begin{abstract}
Study Design: Retrospective chart review.
Purpose: To evaluate the incidence of potential total disc replacement (TDR) candidates among cervical and lumbar fusion patient populations using strict Food and Drug Administration (FDA) criteria and with relative exclusion criteria removed.

Overview of Literature: Recent studies suggest that the potential percentage of patients that are candidates for TDR ranges from $0-5 \%$ in lumbar fusions and $43 \%$ in cervical fusions.
\end{abstract}

Methods: We performed a retrospective chart review of 280 consecutive patients who had lumbar $(n=174)$ and cervical ( $n$ $=106$ ) fusion or TDR performed by one of four independent adult orthopaedic spine surgeons. Charts were screened for investigational device exemption (IDE) inclusion/exclusion criteria and later reanalyzed excluding relative exclusion criteria, such as history of chronic medical illness, twolevel disease (cervical cases), and history of prior fusion surgery in the anatomic region.

Results: Of the 174 lumbar surgeries, 10 were TDR with Prodisc-L and 164 were lumbar fusions. The most common TDR exclusion criteria were lytic spondylolisthesis or spinal stenosis ( $47.7 \%$ of patients) and more than 2 level degenerative disc disease (37.9\%). 14.9\% had no IDE exclusion criteria and would be considered candidates for TDR. After excluding the relative lumbar exclusion criteria, this percentage increased to $25.8 \%$. Of the 106 cervical cases, 3 had a TDR with Prodisc-C and 103 had a cervical fusion. Twenty eight percent had no IDE exclusion criteria and would be considered candidates for cervical TDR.

Conclusions: A larger percentage of cervical fusion candidates are potential candidates for TDR (28\%) than lumbar fusion candidates (14.9\%) based on the strict IDE criteria.

Key Words: Total disc replacement, Incidence, Inclusion/exclusion criteria, Contraindications, Cervical and lumbar fusions

\section{Introduction}

It is estimated that over 325,000 spinal fusions $(137,000$ cervical and 162,000 lumbar) are performed yearly in the US [1]. Clinical interest in the use of total disc replacement (TDR) instead of spinal fusion for the treatment of painful lumbar degenerative disc disease has grown in recent years. In the United States, two lumbar disc prostheses (Charite,
Johnson \& Johnson, LeLocle; Prodisc-L, Synthes, Solothurn, Switzerland) are currently approved for clinical use by the Food and Drug Administration (FDA). There are also two cervical TDR prostheses currently FDA approved for clinical use (Prestige-ST, Medtronic Sofamor Danek, Memphis, TN; Prodisc-C, Synthes). Multiple other lumbar and cervical TDR prostheses are in various stages of clinical trials [2-7]. Several recent reports have documented the clinical efficacy of both lumbar and cervical TDR

Received Mar 9, 2011; 1st Revised Jun 11, 2011; Accepted Jul 14, 2011

Corresponding author: Martin Quirno, MD

155 East 31st Street Apt 20F, New York, NY 10016, USA

Tel: +1-646-724-8970, Fax: +1-212-263-7812, E-mail: mquirno@ gmail.com 


\section{$[2,3,6,8,9]$.}

The theoretical benefit of TDR is to maintain motion in clinical situations where spinal stabilization is indicated. Potentially, this continued motion allows the spine as a whole to function more normally than with fused segments, and it may also limit the additional stresses seen in the motion segments adjacent to fusions, diminishing problems associated with later adjacent segment degeneration. It is generally felt that the increase in TDR procedures over time will result from three forces. One is a growing pool of surgeons trained to perform TDR procedures. The other two forces are an expanded pool of patients willing to undergo the procedure (as opposed to fusion), and an expansion of the possible clinical indications for use beyond the strict

Table 1. Prodisc-L IDE Study Exclusion Criteria

1. DDD of more than one or two diseased levels being treated

2. Known allergy to titanium, polyethylene, cobalt, chromium or molybdenum

3. History of prior fusion surgery at any lumbar level

4. Clinically compromised vertebral bodies at the affected level(s) due to current or past trauma

5. Radiographic confirmation of facet joint disease or degeneration

6. Lytic spondylolisthesis or spinal stenosis

7. Degenerative spondylolisthesis of grade $>1$

8. Back or leg pain of unknown etiology

9. History of osteoporosis (A DEXA bone density measured T score $<-1.0$ ) or history of osteoporosis medications or Osteoporotic fractures in the past

10. History of metabolic bone disease (including Paget's disease and Osteomalacia)

11. History of morbid obesity (defined as a body mass index $>40$ or a weight more than 100 lbs. over ideal body weight)

12. History of any active infection (systemic or local)

13. Known rheumatoid arthritis or other autoimmune disease

14. History of chronic systemic disease including AIDS, HIV, hepatitis

15. History of any active malignancy except non-melanoma skin cancer (minimum of 5 year disease-free interval for any past malignancies treated with curative intent)

IDE: Investigational device exemption, AIDS: Acquired immune deficiency syndrome, HIV: Human immunodeficiency virus.

Table 2. Prodisc-C IDE Study Exclusion Criteria

1. More than one vertebral level being treated

2. Cervical instability on resting lateral or flexion/extension radiographs (translation greater than $3 \mathrm{~mm}$ and/or greater than 11 degrees of rotational difference to that of either adjacent level)

3. Presence of a fused level adjacent to the level to be treated

4. Radiographic confirmation of severe facet joint disease or degeneration

5. Known allergy to cobalt, chromium, molybdenum, titanium or polyethylene

6. Known prior fracture of one or both vertebral levels to be treated

7. Prior surgery at the level to be treated

8. Severe spondylosis at the level to be treated as characterized by any of the following:

- Bridging osteophytes

- Disc height loss greater than $50 \%$

- Absence of motion on flexion-extension radiographs $\left(<2^{\circ}\right)$

9. Neck or arm pain of unknown etiology

10. History of Osteoporosis (A DEXA bone density measured T score < -1.0) or history of osteoporosis medications or Osteoporotic fractures in the past

11. History of metabolic bone disease (including Paget's disease and osteomalacia)

12. Severe diabetes mellitus reuiring daily insulin management

13. History of any active infection (systemic or local)

14. Known rheumatoid arthritis or other autoimmune disease

15. History of chronic systemic disease including AIDS, HIV, Hepatitis

16. History of any active malignancy except non-melanoma skin cancer (minimum of 5 year disease-free interval for any past malignancies treated with curative intent)

IDE: Investigational device exemption, AIDS: Acquired immune deficiency syndrome, HIV: Human immunodeficiency virus. 
FDA study criteria for the initial clinical trials [10-13].

When designing investigational device exemption (IDE) studies for intervertebral disc arthroplasty, a myriad of inclusion and exclusion criteria are enforced in order to strictly define the study patient population and remove potential confounding clinical variables (Tables 1 and 2). Recent series have shown that only $5 \%$ or less of some surgeon's series of lumbar surgical patients had no contraindications to TDR [10]. No previous studies have fully quantified the prevalence of each exclusion criteria for lumbar and cervical TDR.

The purpose of this study was to evaluate a large population of adult spinal fusion patients combined from four independent general adult spine surgical practices to determine the relative incidence of potential TDR candidates among both cervical and lumbar fusion patients using both the strict IDE study criteria as well as potential additional relative indications for use.

\section{Materials and Methods}

We performed a retrospective chart review of 280 consecutive patients who had lumbar $(\mathrm{n}=174)$ and cervical $(\mathrm{n}=$ 106) fusion or TDR procedures performed by four independent adult orthopaedic spine surgeons working within a single academic institution. Three of the four surgeons perform TDR in their practice as investigators in the prospective, randomized cervical and lumbar Prodisc IDE studies. As all TDR patients were assumed to have been candidates for fusion procedures as well, the patients who underwent TDR procedures during this set time interval were also included in the analysis in order to consecutively capture all cervical and lumbar spine fusion candidates who underwent surgery during the period of study. This was necessary in order to arrive at valid incidence data for potential TDR patients among spinal fusion candidates.

Full chart review included examination of demographic data, complete medical history, physical exam, reports of all diagnostic studies, and review of all operative data. This allowed for the complete evaluation of all inclusion and exclusion criteria for the Prodisc cervical and lumbar TDR IDE study protocols for all patients, as well as a complete listing of all of the specific exclusion criteria for which a patient would not be a TDR candidate.

In the secondary analysis, certain exclusion criteria were considered relative rather than absolute and were removed, revealing additional patients which might be TDR candidates (Table 3). Criteria were considered relative if they were not exclusion criteria for IDE trials for other TDR devices. For potential lumbar TDR candidates, this included prior history of lumbar fusion at an adjacent or distant level. For cervical TDR candidates, this included prior history of cervical fusion at an adjacent or distant level, two-level disease (candidate for 2-level TDR), and prior posterior cervical decompression at the index level(s) to be treated. Other criteria considered relative include chronic diseases not affecting the bone quality of the vertebral column and active nonspinal infection (which can be treated prior to TDR), which are common FDA study exclusion criteria in order to limit confounding outcome variables, but are unlikely to specifically affect TDR performance in patients. Significant facet arthrosis, osteoporosis, spinal stenosis, spinal instability (including spondylolysis), spinal deformity, and greater than 2 level disease were still considered absolute contraindications for TDR.

Data analysis was performed using Microsoft Excel 2003 (Microsoft Inc., Redmond, WA, USA). Statistical testing was performed by a two tailed student's $t$-test assuming

Table 3. Exclusion criteria considered relative for cervical and lumbar TDR

\begin{tabular}{ll}
\hline \hline Lumbar TDR & Cervical TDR \\
\hline Prior lumbar fusion & Two level spondylosis/HNP requiring treatment \\
History of metabolic bone disease & Prior cervical fusion \\
History of insulin dependent diabetes & Prior posterior surgery at the treated level \\
History of any active non-spinal infection & History of metabolic bone disease \\
History of RA or other autoimmune disease & History of Insulin dependent diabetes \\
History of chronic disease & History of any active non-spinal infection \\
History of active malignancy & History of RA or other autoimmune disease \\
& History of chronic disease \\
& History of active malignancy \\
\hline
\end{tabular}

TDR: Total disc replacement, RA: Rheumatoid arthritis. 
unequal variances. Statistical significance was defined as $p$ $<0.05$.

\section{Results}

Of a total of 280 patient charts reviewed, 174 were lumbar cases and 106 were cervical. Of the 174 lumbar surgeries, $164(94.5 \%)$ were lumbar fusions and $10(5.3 \%)$ patients underwent TDR. Of the 106 cervical patients, 103 $(97.2 \%)$ underwent cervical fusions and $3(2.8 \%)$ had a TDR. For all patients, $48 \%$ were male and $52 \%$ were female. Mean age for all patients was 51 years. Similar breakdowns were seen for these demographics in the lumbar and cervical subgroups.

Two hundred and twenty eight of the 280 cases had one or more exclusion criteria. For all 280 cases, the average number of TDR exclusion criteria per patient was of 1.6 (range, 0 to 16). For patients with one or more exclusion criteria, the average number of TDR exclusion criteria per patient was 2 (range, 1 to 16). The average number of TDR exclusion criteria per patient with more than one $(\geq 2)$ and two ( $\geq 3$ ) exclusion criteria, was 2.7 and 3.6 respectively. The number of patients excluded for each specific exclusion criteria and the incidence of TDR candidates can be seen in Tables 4 and 5.

In the potential lumbar TDR patient population, the most common TDR exclusion criteria were lytic spondylolisthesis or spinal stenosis ( $47.7 \%$ of patients) followed by more than 2 level degenerative disc disease requiring treatment (37.9\%), and prior lumbar fusion (28.7\%). Of all 174 lumbar cases, $14.9 \%$ (26 patients) had no IDE exclusion criteria and would be considered candidates for TDR. $85.1 \%$ had one or more contraindication. After removing the relative lumbar exclusion criteria, the percentage of potential TDR candidates increased to $25.9 \%$ (45 cases).

Among the potential cervical TDR patients, the most

Table 4. Percentage of patients with the various IDE Exclusion Criteria in lumbar fusion and TDR patients

\begin{tabular}{|c|c|c|c|}
\hline Lumbar Exclusion Criteria & Fusion $(n=164)$ & $\operatorname{TDR}(\mathrm{n}=10)$ & Total $(\mathrm{n}=174)$ \\
\hline DDD greater than 2 levels being treated & 40.2 & 20 & 37.9 \\
\hline Known allergy to prosthetic materials & 1.8 & 0 & 1.7 \\
\hline History of prior lumbar fusion-any level & 30.5 & 0 & 28.7 \\
\hline Vertebral body compromise & 5.5 & 0 & 5.2 \\
\hline Facet joint degeneration & 14.6 & 10 & 13.7 \\
\hline Lytic spondylolisthesis or spinal stenosis & 50.6 & 0 & 47.7 \\
\hline Degenerative spondylolisthesis & 8.53 & 0 & 8.04 \\
\hline Back or leg pain of unknown etiology & 0 & 0 & 0 \\
\hline History of osteoporosis & 3.7 & 0 & 3.4 \\
\hline History of metabolic bone disease & 2.5 & 0 & 2.3 \\
\hline Morbid obesity & 3.65 & 0 & 3.4 \\
\hline History of any active infection & 4.3 & 0 & 4 \\
\hline History of RA or other autoimmune disease & 3.7 & 0 & 3.4 \\
\hline History of chronic disease & 4.3 & 0 & 4 \\
\hline History of active malignancy & 4 & 0 & 4 \\
\hline With one or more exclusion criteria & 88.5 & 30 & 85.1 \\
\hline Without any exclusion criteria & 11.5 & 70 & 14.9 \\
\hline Without absolute exclusion criteria (excluding relative contraindications) & 23.2 & 70 & 25.8 \\
\hline
\end{tabular}

Vaues are presented as percentage.

IDE: Investigational device exemption, TDR: Total disc replacement, RA: Rheumatoid arthritis.

Table 5. Percentage of patients with one or more contraindications

\begin{tabular}{ccccc}
\hline \hline $\begin{array}{c}\text { No. of } \\
\text { contraindications }\end{array}$ & $\begin{array}{c}\text { Lumbar with all } \\
\text { contraindication }\end{array}$ & $\begin{array}{c}\text { Lumbar without } \\
\text { relative contraindications }\end{array}$ & $\begin{array}{c}\text { Cervical with all } \\
\text { contraindication }\end{array}$ & $\begin{array}{c}\text { Cervical without relative } \\
\text { contraindications }\end{array}$ \\
\hline$\geq 1$ & 85 & 74 & 72 & 61 \\
$\geq 2$ & 52 & 19 & 32 & 4 \\
$\geq 3$ & 22 & 5 & 16 & 2 \\
\hline
\end{tabular}

Vaues are presented as percentage. 
common TDR exclusion criteria were greater than one level disease $(45.2 \%)$, severe spondylosis at the treated level (22.7\%), and prior cervical fusion (20.8\%). Of all 106 cervical cases, $28 \%$ (30 cases) had no IDE exclusion criteria and would be considered candidates for cervical TDR. After excluding the relative cervical exclusion criteria, the percentage of potential candidates increased to 39\% (41 cases) (Table 6).

\section{Discussion}

This study found a higher percentage of cervical fusion patients to be candidates for cervical TDR than lumbar fusion patients, both utilizing the strict FDA trial indications and with additional relative indications added. This is not surprising given the current use of and indications for lumbar and cervical fusions. Lumbar fusions done for predominantly back pain due to painful degenerative disc disease, which might be potential TDR cases, represent a small percentage of all lumbar fusions in most general adult spine surgery practices. The majority of lumbar fusions are performed for more advanced degeneration with concomitant facet disease and spinal instability as seen with degenerative spondylolisthesis or scoliosis, and would be absolutely contraindicated for TDR. Cervical fusions done following dis- cectomy to relieve primarily arm pain with or without concomitant neck pain, which might be candidates for cervical TDR, represent a higher percentage of overall cervical fusions. Cervical fusions for isolated neck pain without advanced spondylosis or instability are quite uncommon, and were not an approved indication for TDR in the Prodisc-C IDE trial.

Specific clinical and radiographic factors were considered absolute contraindications for use of cervical and lumbar TDR currently. Osteoporosis (T score $<-1.0$ ) and other metabolic bone diseases will have a higher rate of implant subsidence and vertebral fracture. Prior disc space infection may harbor latent organisms and be at higher risk of infection following TDR. Pain associated with advanced spondylosis with facet joint arthrosis is unlikely to improve following TDR, leaving the facets mobile. Segmental instability, with or without deformity (spondylolisthesis or scoliosis), will place added stress on the implant and may result in early and increased likelihood of failure. Clinically significant lumbar spinal stenosis is commonly associated with advanced facet arthrosis and requires direct decompression. If stabilization is needed, there are usually concomitant advanced degenerative facet changes or segmental instability present.

The exclusion criteria considered relative and examined

Table 6. Percentage of patients with the various IDE exclusion criteria in cervical fusion and TDR patients

\begin{tabular}{|c|c|c|c|}
\hline Cervical Exclusion Criteria & Fusion $(n=103)$ & $\mathrm{TDR}(\mathrm{n}=3)$ & Total $(n=106)$ \\
\hline DDD greater than 1 level being treated & 46.6 & 0 & 45.2 \\
\hline Radiographic cervical instability & 8.7 & 0 & 8.4 \\
\hline Prior adjacent cervical fusion & 20.4 & 0 & 19.8 \\
\hline Facet joint degeneration & 10.7 & 0 & 10.3 \\
\hline Known allergy to prosthetic materials & 0 & 0 & 0 \\
\hline Vertebral body compromise & 9.7 & 0 & 9.4 \\
\hline Prior surgery at the treated level & 10.7 & 0 & 10.3 \\
\hline Severe spondylosis at treated level & 21.4 & 0 & 20.8 \\
\hline Neck or arm pain of unknown etiology & 0 & 0 & 0 \\
\hline History of osteoporosis & 1.9 & 0 & 1.9 \\
\hline History of metabolic bone disease & 0 & 0 & 0 \\
\hline History of insulin dependent diabetes & 0 & 0 & 0 \\
\hline History of any active infection & 1.9 & 0 & 1.9 \\
\hline History of RA or other autoimmune disease & 5.8 & 0 & 5.7 \\
\hline History of chronic disease & 1.9 & 0 & 1.9 \\
\hline History of active malignancy & 0.7 & 0 & 0.9 \\
\hline With one or more exclusion criteria & 74 & 0 & 72 \\
\hline Without any exclusion criteria & 26 & 100 & 28 \\
\hline Without absolute exclusion criteria (excluding relative contraindications) & 37 & 100 & 39 \\
\hline
\end{tabular}

Vaues are presented as percentage.

IDE, investigational device exemption; TDR, total disc replacement; RA, rheumatoid arthritis. 
as potential new indications were chosen based on both the differing inclusion criteria of other current TDR IDE trials as well as the real world practice of medicine outside of clinical trials. Patients with a history of chronic illness such as insulin dependent diabetes, rheumatoid arthritis, and other autoimmune disease, are excluded from FDA trials but may otherwise be excellent candidates for TDR following a thorough assessment of bone quality. Patients with active non-spinal infection or malignancy may be TDR candidates, all other things considered, once their active nonspine disease is treated and adequately controlled. Twolevel lumbar disease was included in the IDE study and kept as part of the lumbar inclusion criteria for this analysis despite the fact that, as of the time of the writing of this manuscript, the two-level data has not been fully reviewed by the FDA and so this is not yet an approved indication. Two-level disease, common in the cervical spine, was not included in the FDA IDE trial, so it was considered a relative indication. TDR next to a prior adjacent or nonadjacent fusion was an exclusion criteria for both of the Prodisc IDE trials. However, it is an acceptable inclusion criteria for other cervical and lumbar TDR IDE trials, and so it was considered a relative indication for this analysis. Prior posterior decompressive surgery at the index level was considered an exclusion criterion for the Prodisc-C IDE trial but not for the Prodisc-L trial, so it was made a relative indication for the cervical patients in the secondary analysis.

Other studies looking at the potential incidence of TDR candidates have provided varying results. Huang et al. [10], reviewing 100 consecutive lumbar surgical procedures, found only $5 \%$ of patients without contraindications for TDR.2 This series, however, also included 44 patients who underwent nonfusion surgical procedures including decompressions (in 42 of the patients), IDET (4 patients), and lumbar TDR (1). All of their fusion patients (100\%) also underwent spinal canal decompressions, so no fusion cases were done for back pain only. Although none of their fusions cases had no contraindications for TDR, if the TDR case and 4 IDET cases are included (which should have no TDR contraindications), then the true number of fusionindicated cases rises to 61 and the number of potential TDR candidates in that group rises to 5 , or $8 \%$ of 'possible' fusion cases.

Wong et al. [14], reviewing 100 consecutive 1-3 level lumbar fusions, found at least one contraindication in every patient (with an average number of 3.64 contraindications per patient), leaving none as potential TDR candidates.
Facet disease was noted as a contraindication in 97 patients, but was only felt to be definitely clinically significant in 9 . Seventy-five of the cases had fusion surgery for spondylolisthesis, 31 cases had a herniated disc with radiculopathy, 15 cases had scoliosis, 16 had deficient posterior elements, and 2 were done for pseudoarthrosis. It is likely, given the contraindications reported, that few if any of the reviewed fusions performed by these 5 spine surgeons ( 3 orthopedic, 2 neurological) were performed for the diagnosis of discogenic low back pain.

Chin [15] presented a similar study in which he analyzed the presence of the FDA exclusion/inclusion criteria on potential surgical candidates for lumbar spine surgery. He concludes that there was only a $0.5 \%$ incidence of indications for TDR in the overall population screened (627 patients). It is important to point out that only $21 \%$ of these patients were candidates for surgery, and that only $9 \%$ had a spinal fusion. When analyzing the data on his fusion patients, he reports a 5\% incidence of no contraindications. This study does not describe how the clinical significance of each contraindication may interfere with the incidence reported.

Auerbach et al. [16] reported a $43 \%$ incidence of no contraindications to cervical TDR using the current IDE inclusion and exclusion criteria from 4 different cervical TDR IDE trials. They also found that an additional $4.2 \%$ of patients would have qualified as TDR candidates if treatment for adjacent segment disease was included.

There are a variety of factors with have limited the widespread acceptance of lumbar TDR to date. Insurance authorization for the procedure has been very limited, often only on a case by case basis. Even those cases where insurance company authorization is possible are often approved only for TDR within the strict FDA guidelines established by the randomized control trial. Some have called for more longterm data, which is not yet available in the United States patient population. Finally, surgeons remain concerned over the implant longevity and the difficulty with anterior revision procedures, if needed, especially for the lumbar spine.

\section{Conclusions}

In our consecutive series of lumbar and cervical fusion surgical candidate patients, a larger percentage of cervical fusion candidates were found to be potential candidates for TDR (28\%) than lumbar fusion candidates (14.9\%) based on the strict IDE study inclusion and exclusion criteria. 
When potential relative contraindications are removed, the percentage of potential TDR candidates rose in both groups, with the percentage of cervical patients remaining higher than lumbar patients (39\% vs. $25.8 \%$ ).

\section{REFERENCES}

1. National Center for Health Statistics, Centers for Disease Control and Prevention. 2003 National Hospital Discharge Survey [Internet]. Rosemont: American Academy of Orthopaedic Surgeons, [Cited 2011 Mar 1]. Available from: http://www.aaos. org.

2. Delamarter RB, Bae HW, Pradhan BB. Clinical results of ProDisc-II lumbar total disc replacement: report from the United States clinical trial. Orthop Clin North Am 2005; 36:301-13.

3. McAfee PC, Cunningham B, Holsapple G, et al. A prospective, randomized, multicenter Food and Drug Administration investigational device exemption study of lumbar total disc replacement with the CHARITE artificial disc versus lumbar fusion: part II: evaluation of radiographic outcomes and correlation of surgical technique accuracy with clinical outcomes. Spine (Phila Pa 1976) 2005;30: 1576-83.

4. Mathews HH, Lehuec JC, Friesem T, Zdeblick T, Eisermann L. Design rationale and biomechanics of Maverick Total Disc arthroplasty with early clinical results. Spine J 2004;4(6 Suppl):268S-275S.

5. Coric D, Finger F, Boltes P. Prospective randomized controlled study of the Bryan Cervical Disc: early clinical results from a single investigational site. J Neurosurg Spine 2006;4:31-5.

6. Pracyk JB, Traynelis VC. Treatment of the painful motion segment: cervical arthroplasty. Spine (Phila Pa 1976) 2005;30(16 Suppl):S23-32.

7. Errico TJ. Lumbar disc arthroplasty. Clin Orthop Relat Res
2005;(435):106-17.

8. Zigler J, Delamarter R, Spivak JM, et al. Results of the prospective, randomized, multicenter Food and Drug Administration investigational device exemption study of the ProDisc-L total disc replacement versus circumferential fusion for the treatment of 1-level degenerative disc disease. Spine (Phila Pa 1976) 2007;32:1155-62.

9. Stachniak J, Sawin P, Mummaneni P, Ceola W, Zdeblick T. Prestige ST cervical disc IDE study: five site pooled data. Spine J 2006;6(5 Suppl):18S-19S.

10. Huang RC, Lim MR, Girardi FP, Cammisa FP Jr. The prevalence of contraindications to total disc replacement in a cohort of lumbar surgical patients. Spine (Phila Pa 1976) 2004;29:2538-41.

11. Bertagnoli R, Kumar S. Indications for full prosthetic disc arthroplasty: a correlation of clinical outcome against a variety of indications. Eur Spine J 2002;11 Suppl 2:S131-6.

12. Kim WJ, Lim ST, Lee SH. Artificial disc replacement $(\mathrm{ADR})$ as an alternative for spinal fusion in the treatment of lumbar spinal stenosis: two-year results of a prospective study. 5th Annual Global Symposium Spine Arthroplasty Society; 2005 May 3-6; New York, USA.

13. Tay BB, Berven S. Indications, techniques, and complications of lumbar interbody fusion. Semin Neurol 2002;22:221-30.

14. Wong DA, Annesser B, Birney T, et al. Incidence of contraindications to total disc arthroplasty: a retrospective review of 100 consecutive fusion patients with a specific analysis of facet arthrosis. Spine J 2007;7:5-11.

15. Chin KR. Epidemiology of indications and contraindications to total disc replacement in an academic practice. Spine J 2007;7:392-8.

16. Auerbach JD, Jones KJ, Fras CI, Balderston JR, Rushton SA, Chin KR. The prevalence of indications and contraindications to cervical total disc replacement. Spine J 2008;8:711-6. 